\title{
'Critical View of Safety' (CVS) as an Alternative to Routine Intraoperative Cholangiography (IOC) During Laparoscopic Cholecystectomy for Acute Biliary Pathology
}

\author{
Pandanaboyana Sanjay • David J. Exon
}

Received: 12 December 2010/Accepted: 2 February 2011 /Published online: 19 February 2011

(C) 2011 The Society for Surgery of the Alimentary Tract

\section{Dear Editor}

We would like to thank Buddingh et al. for their interest and comments on our article. We adopted the policy of selective intraoperative cholangiography (IOC), with its use restricted to patients with increased risk of ductal stones. In our opinion, the indications for IOC are twofold-firstly, to clarify the anatomy on the Calot's triangle and, secondly, to exclude common bile duct (CBD) stones. In the presence of normal liver function tests and a non-dilated duct, the risk of harbouring CBD stones is extremely low, and a critical view of safety in the majority of the cases clarifies the anatomy, thereby excluding anatomical variations in the Calot's triangle. We do not advocate 'critical view of safety' (CVS) as an alternative to IOC in the presence of anatomical variations confirmed either on preoperative imaging or noted intraoperatively. The size of the current series precluded identification of such anatomical variations.

Advocates for routine IOC during laparoscopic cholecystectomy claim that IOC reduces biliary injury and allows for the diagnosis of asymptomatic choledocholithiasis. In order for IOC to satisfy these assertions, operators need to be able to both perform and correctly interpret the IOC which can be technical and interpretative. ${ }^{1}$ In our experience, from a recent survey of surgeons in New Zealand (unpublished data), accuracy of identifying anatomical variations during IOC was poor irrespective of policy of IOC (routine versus selective). This raises questions regarding training in IOC and the appropriateness of routinely performing IOC. This is probably reflected in the current practice of majority of the surgeons both in the UK and New Zealand who practice a policy of selective IOC. ${ }^{2}$

We agree with Buddingh et al. that IOC is an important technique in the surgeons' armamentarium to minimise the risk of bile duct injury. Nevertheless, it carries the inherent risk of misinterpretation of anatomy and identification of asymptomatic choledocholithiasis necessitating further invasive investigations. We therefore recommend a selective policy of IOC in the presence of high risk of CBD stones or aberrant anatomy noted whilst obtaining CVS.

\section{References}

1. Turner M. Cho S, Messmer JM. Pitfalls in cholangiographic interpretation. Radiographics 1987;7:1067-1105

2. Sanjay P, Kulli C, Polignano FM, Tait IS. Optimal surgical technique, use of intraoperative cholangiography, and management of acute gallbladder disease. Results of a nationwide survey in the UK and Ireland. Annals of Royal College of Surgeons of England 2010;92:302-306.
P. Sanjay $(\bowtie) \cdot$ D. J. Exon

Ninewells Hospital and Medical School,

Dundee, UK

e-mail: sanjay.pandanaboyana@nhs.net 